\title{
Valvoplastia por Via Carotídea na Estenose Aórtica do Neonato e Lactente Jovem: Resultados Imediatos em Serviços de Referência
}

\author{
Rodrigo Nieckel da Costa ${ }^{1}$, Valmir Fernandes Fontes ${ }^{1,2}$, Simone Rolim Fernandes Fontes Pedra ${ }^{1,2}$, \\ Magali Arraes dos Santos ${ }^{1,2}$, Samuel Martins Moreira', Maria Virgínia Tavares Santana', \\ leda Biscegli Jatene ${ }^{2}$, Patrícia Figueiredo², César Augusto Esteves ${ }^{1}$, \\ Sérgio Luiz Navarro Braga ${ }^{1}$, Carlos Augusto Cardoso Pedra ${ }^{1,2}$
}

\section{RESUMO}

Introdução: A valvoplastia aórtica por cateter-balão em neonatos e lactentes ainda é um procedimento discutido na literatura. Neste artigo são relatados os resultados imediatos desse procedimento realizado pela via carotídea. Método: Estudo longitudinal de uma coorte de pacientes com estenose valvar aórtica grave ou crítica congênita com até seis meses de idade, nos quais foi realizada valvuloplastia aórtica com cateter-balão no período de 1997 a 2008. Resultados: Entre março de 1997 e março de 2008, foram realizados 25 procedimentos em 24 pacientes $(79 \%$ do sexo masculino), com média de idade de 1,4 \pm 1 mês (0,1-4 meses) e média de peso de $3,7 \pm 1,2 \mathrm{~kg}(1,1-5,2 \mathrm{~kg})$. A relação balão-anel foi de $0,93 \pm 0,1$. Óbitos relacionados ao procedimento ocorreram em 4 pacientes $(17 \%)$, três por falência ventricular esquerda e um por dissecção do seio de Valsalva da artéria coronária esquerda durante o posicionamento do guia e introdutor antes da realização da valvoplastia. O gradiente sistólico máximo transvalvar à ecocardiografia caiu de $86 \pm 25 \mathrm{mmHg}$ para $36 \pm 15 \mathrm{mmHg}$, enquanto o gradiente pico-a-pico caiu de $61 \pm 36 \mathrm{mmHg}$ para $29 \pm 17 \mathrm{mmHg}$ após o procedimento. Melhora significativa da função ventricular foi observada nos pacientes com disfunção prévia. Houve aparecimento ou piora de insuficiência aórtica em 10 pacientes, não sendo necessária intervenção cirúrgica imediata. Fibrilação ou taquicardia ventricular foi observada em 4 pacientes. No seguimento de 7 pacientes, 2 necessitaram de cirurgia valvar. Conclusões: Embora ainda seja um procedimento associado a morbidade e mortalidade significativas, a valvoplastia aórtica é eficaz na redução do gradiente transvalvar e na preservação da função ventricular e é capaz de postergar a intervenção cirúrgica.

DESCRITORES: Estenose da valva aórtica/cirurgia. Angioplastia transluminal percutânea coronária. Procedimentos cirúrgicos cardíacos. Ecocardiografia. Recém-nascido. Lactente. Adolescente.

\footnotetext{
1 Instituto Dante Pazzanese de Cardiologia - São Paulo, SP, Brasil. 2 Hospital do Coração da Associação do Sanatório Sírio - São Paulo, SP, Brasil.

Correspondência: Carlos A. C. Pedra. Instituto Dante Pazzanese de Cardiologia. Av. Dr. Dante Pazzanese, 500 - Ibirapuera - São Paulo, SP, Brasil - CEP 04012-180

E-mail: cacpedra@uol.com.br

Recebido em: 29/9/2009 • Aceito em: 17/11/2009
}

\section{ABSTRACT}

Aortic Valvuloplasty Using the Carotid Approach in Neonates and Infants with Aortic Stenosis: Immediate Outcomes in Reference Centers

Background: Balloon aortic valvuloplasty still remains a debatable procedure in the literature. In this paper, we report the immediate results of this procedure using the carotid access. Method: Longitudinal study of a cohort of patients with severe or critical congenital aortic valvular stenosis with up to six months of age treated with balloon aortic valvuloplasty from 1997 to 2008. Results: Between March 1997 and March 2008, 25 procedures were performed in 24 patients $(79 \%$ males $)$ with mean age of $1.4 \pm 1.0$ months (0.1-4 months) and mean weight of $3.7 \pm 1.2 \mathrm{~kg}(1.1-5.2 \mathrm{~kg})$. The balloon-annulus ratio was $0.93 \pm 0.1$. There were 4 procedure-related deaths $(17 \%)$, three of them due to left ventricular dysfunction and one due to the dissection of the left sinus of Valsalva during positioning of the guide wire and sheath prior valvuloplasty. The peak transvalvar systolic gradient at echocardiography decreased from $86 \pm 25 \mathrm{mmHg}$ to $36 \pm 15 \mathrm{mmHg}$ whereas the peak-to-peak gradient decreased from $61 \pm 36 \mathrm{mmHg}$ to $29 \pm 17 \mathrm{mmHg}$ after the procedure. There was significant improvement of ventricular function in patients with previous dysfunction. There was worsening or de-novo aortic failure in 10 patients, which did not require immediate surgical intervention. Ventricular tachycardia or ventricular fibrillation was observed in 4 patients. During follow-up, valve surgery was required in 2 of 7 patients. Conclusions: Although still associated to significant morbidity and mortality, balloon aortic valvuloplasty is effective in reducing transvalvar gradient and in preserving left ventricular function and may delay the need for surgical intervention.

DESCRIPTORS: Aortic valve stenosis/surgery. Angioplasty, transluminal, percutaneous coronary/methods. Cardiac surgical procedures. Echocardiography. Infant, newborn. Neonate. Infant. Adolescent. 
E tenose aórtica valvar grave é definida como toda estenose localizada no plano valvar, em que o gradiente sistólico máximo por ecocardiografia for superior a cerca de $70 \mathrm{mmHg}^{1-4}$. Estenose aórtica valvar crítica é aquela que se manifesta clinicamente no neonato e depende da manutenção do canal arterial e, consequentemente, da função de bomba do ventrículo direito para manter débito cardíaco sistêmico. Essa situação representa a forma mais grave da doença, pelas características morfológicas que, por vezes, se sobrepõem àquelas da síndrome de hipoplasia de ventrículo esquerdo $^{1-5}$. Estima-se que menos de $3 \%$ dos neonatos com cardiopatias congênitas sejam portadores de estenose aórtica valvar crítica ${ }^{2}$. O neonato com estenose aórtica valvar grave ou crítica pode se apresentar com comprometimento hemodinâmico significativo, com falência ventricular esquerda e sinais de baixo débito cardíaco necessitando de intervenção precoce para evitar maior deterioração de seu estado clínico ${ }^{1,2,6}$. O tratamento desses pacientes de alto risco pode ser realizado por intervenção cirúrgica ou percutânea, sendo esta última amplamente aceita e utilizada como método terapêutico paliativo inicial nos pacientes com indicação de algoritmo biventricular no período neonatal ${ }^{7}$. Entretanto, a morbidade e a mortalidade associadas ao procedimento ainda são elevadas, oscilando entre 5\% e $20 \%$ em diversas séries, inclusive nas mais contemporâneas ${ }^{8-10}$. Na literatura nacional, encontram-se dados escassos sobre os desfechos imediatos da valvoplastia aórtica com cateter-balão. Nosso objetivo, neste artigo, é relatar os resultados imediatos desse tipo de intervenção realizada por via carotídea em dois hospitais cardiológicos de referência na cidade de São Paulo.

\section{MÉTODO}

\section{Planejamento do estudo}

Estudo longitudinal de uma coorte de pacientes com estenose valvar aórtica grave ou crítica congênita, na qual foi realizada valvuloplastia aórtica com cateter-balão no período de 1997 a 2008 em dois hospitais cardiológicos de referência na cidade de São Paulo. O levantamento dos dados foi realizado de modo retrospectivo. Este estudo foi aprovado pela Comissão de Ética de ambos os hospitais.

\section{Critérios de seleção}

Foram incluídos neste estudo todos os neonatos e lactentes jovens até seis meses de idade portadores de estenose aórtica valvar crítica ou grave submetidos a valvoplastia aórtica por cateter-balão. Foram considerados portadores de estenose aórtica crítica todos os dependentes do canal arterial para manter o débito cardíaco sistêmico e portadores de estenose aórtica valvar grave aqueles com gradiente sistólico máximo à ecocardiografia acima de $70-80 \mathrm{mmHg}$ e/ou gradiente pico-apico acima de $50 \mathrm{mmHg}$ pelo cateterismo. Também foram incluídos neste estudo pacientes com gradientes mais baixos, porém com sinais de disfunção ventricular esquerda e baixo débito. Foram excluídos os pacientes encaminhados para algoritmo univentricular após avaliação pré-procedimento por ecocardiografia (conforme descrito a seguir) e os pacientes em que o grau de insuficiência aórtica foi considerado igual ou maior que moderado.

\section{Avaliação pré-procedimento}

Antes do procedimento foi realizada avaliação criteriosa dos pacientes selecionados para intervenção, com exame clínico, radiografia de tórax, eletrocardiograma e ecocardiografia transtorácica. Na ecocardiografia foram avaliados diversos aspectos, tais como anatomia da válvula, fração de ejeção do ventrículo esquerdo, tamanho e divisão desse ventrículo (via de entrada, via de saída e corpo), bem como diâmetros do anel mitral e aórtico.

\section{Procedimento}

Todos os procedimentos foram realizados sob anestesia geral endovenosa. Em todos os pacientes a via carotídea foi utilizada para o acesso arterial. No primeiro paciente da casuística foi realizada punção percutânea da carótida comum direita e no restante a carótida comum foi dissecada por equipe de cirurgia vascular ou cardíaca seguida de punção sob visualização direta, conforme já descrito previamente ${ }^{8,11}$. Após punção sob visualização direta, foi avançado fio-guia para a aorta ascendente com subsequente posicionamento de introdutor $5 \mathrm{~F}$ ou $6 \mathrm{~F}$ para realização do procedimento. Utilizou-se heparina na dose de $100 \mathrm{U} / \mathrm{kg}$ a $150 \mathrm{U} / \mathrm{kg}$ após a obtenção do acesso arterial. Os pacientes foram rapidamente estudados e os procedimentos, realizados segundo a técnica clássica descrita. Em casos mais graves, os pacientes foram poupados da ventriculografia (e às vezes até mesmo da aortografia), sendo realizada a dilatação de imediato. Após as medidas pressóricas e as angiografias, a valva aórtica foi cruzada de forma retrógrada com o uso de cateteres de furo terminal e fio-guia de coronária 0,014 polegada, levando em consideração a anatomia tanto da aorta ascendente como da valva aórtica (Figura 1). Após o fio-guia atingir o ventrículo esquerdo, o cateter foi avançado para realização da manometria, quando a condição clínica do paciente permitia. Se houvesse arritmia ou instabilidade hemodinâmica importante procedia-se à valvoplastia sem medidas pressóricas prévias, conforme já mencionado anteriormente. Por vezes, o fio-guia 0,014 polegada foi trocado por fioguia de troca 0,035 polegada, que era previamente moldado com uma grande alça em sua extremidade para proteção do miocárdio do ventrículo esquerdo (Figura 2). Sobre ele foi avançado e posicionado, no nível do anel valvar, cateter-balão para realização da dilatação (Figura 3). O balão foi inicialmente escolhido de forma a que a relação balão-anel valvar aórtico fosse igual a 0,8-0,9. Se não houvesse queda significativa do gradiente pressórico para valores pico-a-pico abaixo de $40 \mathrm{mmHg}$, procedia-se a nova valvoplastia 
Costa RN, et al. Valvoplastia por Via Carotídea na Estenose Aórtica do Neonato e Lactente Jovem: Resultados Imediatos em Serviços de Referência. Rev Bras Cardiol Invas. 2009;17(4):526-32.

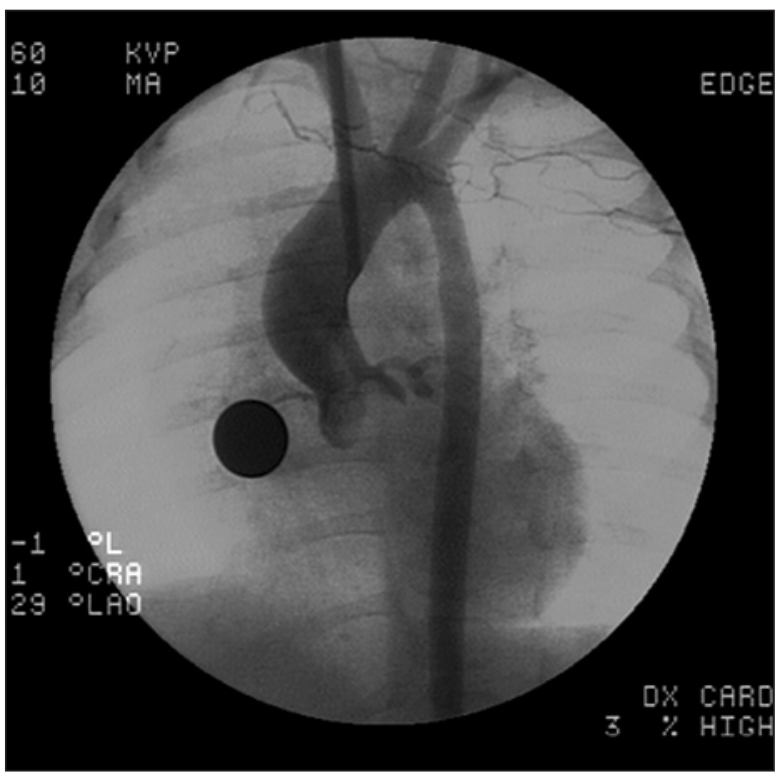

Figura 1 - Anel valvar aórtico e aorta ascendente.

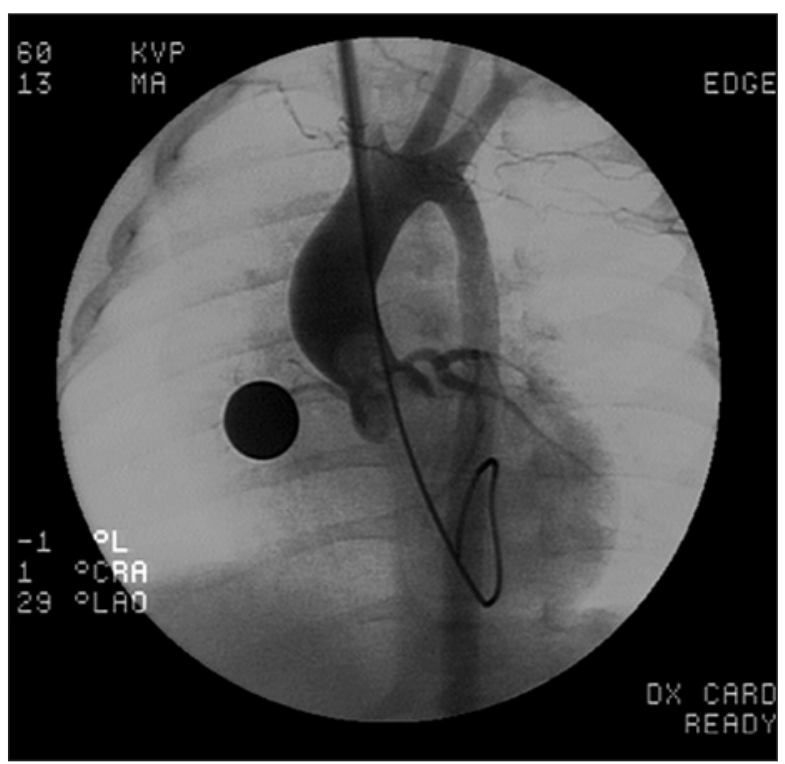

Figura 2 - Fio-guia previamente moldado.

com balão 1 mm maior (se não houvesse insuficiência aórtica acima de moderada) até que fosse obtido resultado satisfatório. Foram utilizados balões Tyshak (NuMED Inc., Hopkinton, Estados Unidos) e Powerflex (Johnson \& Johnson-Cordis Corp., Miami Lakes, Estados Unidos) e alguns balões de angioplastia periférica, todos materiais normalmente registrados pela Agência Nacional de Vigilância Sanitária (Anvisa). Para manter a estabilidade do balão, avançou-se o introdutor arterial de forma a que sua porção distal ficasse em contato com o cateter-balão, promovendo, assim, maior estabilidade no momento da insuflação. Após o pro-

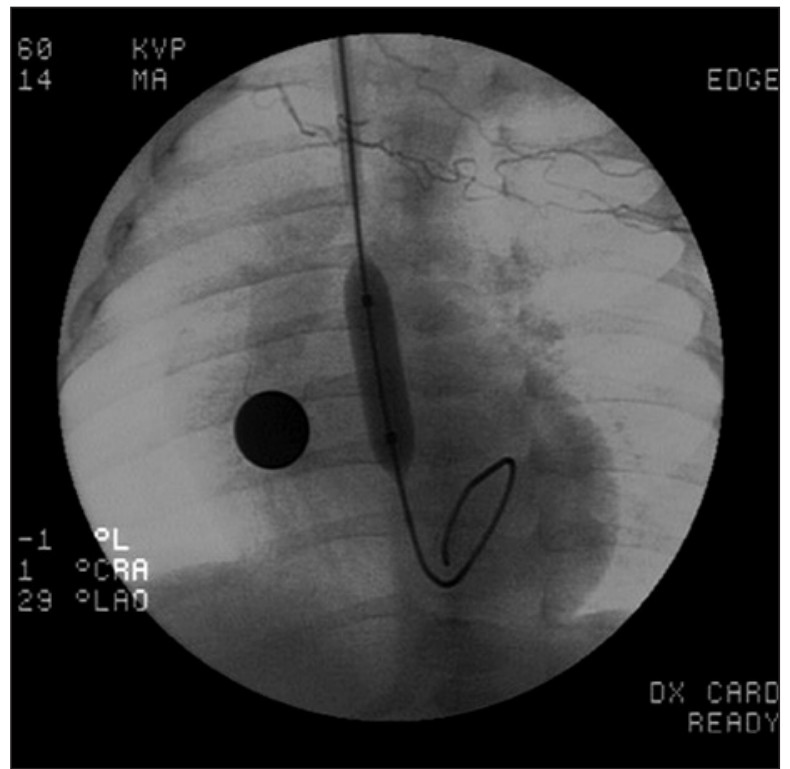

Figura 3 - Cateter-balão no nível do anel valvar aórtico.

cedimento, quando possível, foram realizadas novas angiografias e medidas pressóricas. O sucesso do procedimento foi definido pela queda do gradiente transvalvar de pelo menos $50 \%$ e para menos de $40 \mathrm{mmHg}$ na ausência de insuficiência aórtica grave. Após o procedimento foi realizada rafia da artéria carótida com pontos separados até atingir hemostasia e revertida $50 \%$ da dose utilizada de heparina.

Os pacientes foram então transferidos para a Unidade de Terapia Intensiva (UTI) para recuperação pósprocedimento. Durante a internação na UTI foram realizados ecocardiografia, radiografia de tórax e eletrocardiograma de controle. Após a alta hospitalar os pacientes foram acompanhados pelo cardiologista pediátrico de referência nos hospitais em que foram realizados os procedimentos ou em sua cidade de origem. Durante o seguimento, foram avaliados por ecocardiografia, radiografia de tórax e eletrocardiograma. Não foram realizados exames para avaliação da patência da carótida (Doppler ou ressonância magnética) de rotina.

\section{Análise estatística}

Os valores estão expressos em médias com desvio padrão e/ou medianas e variação conforme a distribuição da amostra. O teste de qui-quadrado foi utilizado para comparar variáveis qualitativas e o teste $t$ de Student, para variáveis quantitativas antes e depois do procedimento. O nível de significância de alfa foi de 5\%.

\section{RESULTADOS}

\section{Pacientes}

No período entre março de 1997 e março de 2008, foram realizados 25 procedimentos em 24 pacientes, 
Costa RN, et al. Valvoplastia por Via Carotídea na Estenose Aórtica do Neonato e Lactente Jovem: Resultados Imediatos em Serviços de Referência. Rev Bras Cardiol Invas. 2009;17(4):526-32.

sendo 19 do sexo masculino (79\%). A média de idade foi de 1,4 \pm 1 mês $(0,1-4$ meses $)$ e a média de peso foi de $3,7 \pm 1,2 \mathrm{~kg}(1,1-5,2 \mathrm{~kg}$ ) (Tabela 1). A valva foi avaliada por ecocardiografia como verdadeiramente bicúspide em 7 pacientes (30\%). Em todos os pacientes a carótida comum foi considerada de grande tamanho e apropriada para a introdução das bainhas. Óbitos relacionados ao procedimento ocorreram em 4 pacientes $(17 \%)$, três deles decorrentes de falência ventricular esquerda e um por dissecção do seio de Valsalva da artéria coronária esquerda durante o posicionamento do guia e introdutor antes da realização da valvoplastia. Um neonato faleceu oito dias após o procedimento, em decorrência de broncoaspiração. Quatro dos cinco óbitos ocorreram nos primeiros seis anos de nossa casuística. O anel valvar teve média de 7,3 $\pm 1,0 \mathrm{~mm}(6-9 \mathrm{~mm})$ à ecocardiografia e $8,1 \pm 1,3 \mathrm{~mm}(6-10 \mathrm{~mm})$ no cateterismo. Os balões utilizados tiveram diâmetro médio de 7,6 \pm 1,4 mm (6-12 mm), estabelecendo, dessa forma, relação balão-anel igual a $0,93 \pm 0,1$. A função ventricular esquerda antes do procedimento foi considerada moderada ou gravemente comprometida em 9 pacientes $(37 \%)$, sendo normal ou discretamente reduzida nos outros 15 pacientes (63\%). O gradiente sistólico máximo transvalvar por ecocardiograma prévio ao procedimento foi de $86 \pm 25 \mathrm{mmHg}(49-135 \mathrm{mmHg})$, enquanto o gradiente pico-a-pico no laboratório de hemodinâmica foi de $61 \pm 36 \mathrm{mmHg}$.

\section{Resultados imediatos}

Em um paciente, optou-se por repetir o procedimento dois dias após o primeiro, utilizando-se balões de diâmetro maior que não estavam disponíveis então. Após o procedimento, a ecocardiografia demonstrou melhora significativa da função ventricular na maioria dos casos, com 75\% deles (18 pacientes) apresentando função normal ou discretamente reduzida. Apenas em $2(8 \%)$ a função foi considerada moderadamente reduzida $(P<0,05)$. Houve redução do gradiente sistólico

TABELA 1

Características dos pacientes e dados técnicos do procedimento

\begin{tabular}{lc}
\hline ldade, média $\pm \mathrm{DP}$ & $1,4 \pm 1$ mês $(0,1-4$ meses $)$ \\
$\begin{array}{l}\text { Peso, média } \pm \mathrm{DP} \\
\text { Sexo masculino, } \mathrm{n}\end{array}$ & $3,7 \pm 1,2 \mathrm{~kg}(1,1-5,2 \mathrm{~kg})$ \\
$\begin{array}{l}\text { Anel valvar aórtico ao } \\
\text { ecocardiograma, média } \pm \mathrm{DP}\end{array}$ & $7,3 \pm 1 \mathrm{~mm}(6-9 \mathrm{~mm})$ \\
$\begin{array}{l}\text { Anel valvar aórtico à } \\
\text { hemodinâmica, média } \pm \mathrm{DP}\end{array}$ & $8,1 \pm 1,3 \mathrm{~mm}(6-10 \mathrm{~mm})$ \\
$\begin{array}{l}\text { Balão utilizado, média } \pm \mathrm{DP} \\
\text { Relação balão-anel, média } \pm \text { DP }\end{array}$ & $7,6 \pm 1,4 \mathrm{~mm}(6-12 \mathrm{~mm})$ \\
\hline DP = desvio padrão; $\mathrm{n}=$ número de pacientes.
\end{tabular}

máximo à ecocardiografia para $36 \pm 15 \mathrm{mmHg}$ no exame entre 24 horas e 5 dias pós-dilatação $(P<0,001)$. O gradiente na sala de hemodinâmica caiu para $29 \pm 17 \mathrm{mmHg}$ no pós-procedimento. Os dados hemodinâmicos e ecocardiográficos estão apresentados na Tabela 2. Insuficiência valvar aórtica discreta foi descrita em um paciente previamente ao procedimento. Após o procedimento houve incremento do grau de insuficiência aórtica no grupo como um todo $(P<0,05)$, sendo considerada mínima ou discreta em 7 pacientes $(30 \%)$, moderada em 2 pacientes $(8 \%)$ e grave em 1 paciente (4\%) (Figura 4). O paciente com insuficiência aórtica prévia ao procedimento não apresentou incremento no grau de insuficiência à ecocardiografia pósprocedimento. Em nenhum dos pacientes foi necessária intervenção cirúrgica imediata relacionada à insuficiência aórtica. Outras complicações relacionadas ao procedimento foram encontradas em 9 pacientes (37\%). No primeiro paciente desta série, a via de acesso arterial foi através de punção carotídea, conforme já descrito. A punção foi difícil e após o procedimento o paciente apresentou convulsões tônico-clônicas, provavelmente decorrentes de embolia aérea durante a punção. O paciente teve diagnóstico de síndrome de Horner e em seu acompanhamento neurológico evoluiu com exames tomográficos e eletroencefalograma normais. Após essa complicação optou-se por dissecção e punção carotídea sob visualização direta. Em 1 paciente ocorreu sangramento no local da dissecção carotídea com necessidade de revisão cirúrgica, que foi realizada na UTI e não necessitou transfusão sanguínea. Em todos os pacientes ocorreram extrassístoles ventriculares à entrada no ventrículo esquerdo e em 4 pacientes $(16 \%)$ ocorreram arritmias ventriculares (3 com fibrilação ventricular e 1 com taquicardia ventricular). Nos 4 últimos, o distúrbio elétrico foi revertido após realização de manobras de cardioversão elétrica e/ou drogas.

\section{Seguimento}

Desta amostragem, o acesso às consultas de seguimento só foi possível em 7 pacientes. Desses pacientes, 4 seguem em acompanhamento clínico sem necessidade de reintervenção, assintomáticos e com insuficiência aórtica considerada leve ou moderada; 1 paciente realizou nova valvoplastia com balão seis anos após o procedimento e em sua evolução necessitou cirurgia para troca valvar sem intercorrências; e outro paciente em que foi necessária intervenção cirúrgica realizou procedimento de Ross com quatro anos de evolução, também sem intercorrências.

\section{DISCUSSÃO}

A valvoplastia aórtica percutânea na criança e no adulto jovem vem sendo o método de escolha para o tratamento paliativo inicial da estenose aórtica. Entretanto, essa modalidade de intervenção ainda segue sendo discutida quando o paciente se encontra nos 
Costa RN, et al. Valvoplastia por Via Carotídea na Estenose Aórtica do Neonato e Lactente Jovem: Resultados Imediatos em Serviços de Referência. Rev Bras Cardiol Invas. 2009;17(4):526-32.

TABELA 2

Dados hemodinâmicos da valvoplastia

\begin{tabular}{lccc}
\hline & $\begin{array}{c}\text { Pré-procedimento } \\
\text { (média } \pm \text { DP) }\end{array}$ & $\begin{array}{c}\text { Pós-procedimento } \\
\text { (média } \pm \text { DP) }\end{array}$ & P \\
\hline Pressão sistólica na aorta & $50 \pm 11 \mathrm{mmHg}$ & $71 \pm 19 \mathrm{mmHg}$ & $<0,001$ \\
Pressão diastólica na aorta & $32 \pm 10 \mathrm{mmHg}$ & $39 \pm 12 \mathrm{mmHg}$ & 0,14 \\
Pressão sistólica no ventrículo esquerdo & $111 \pm 35 \mathrm{mmHg}$ & $100 \pm 25 \mathrm{mmHg}$ & 0,19 \\
Pressão diastólica no ventrículo esquerdo & $25 \pm 8 \mathrm{mmHg}$ & $15 \pm 11 \mathrm{mmHg}$ & $<0,05$ \\
Gradiente sistólico à hemodinâmica & $61 \pm 36 \mathrm{mmHg}$ & $29 \pm 17 \mathrm{mmHg}$ & $<0,001$ \\
Gradiente sistólico à ecocardiografia & $86 \pm 25 \mathrm{mmHg}$ & $36 \pm 15 \mathrm{mmHg}$ & $<0,001$ \\
\hline DP $=$ desvio padrão. & & &
\end{tabular}

$\mathrm{DP}=$ desvio padrão

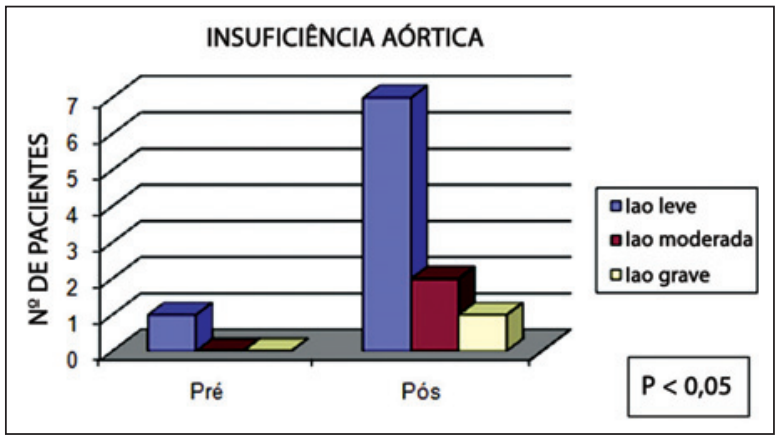

Figura 4 - Progressão de insuficiência aórtica pós-procedimento. lao = insuficiência aórtica.

primeiros meses de vida, em decorrência dos questionamentos sobre morbidade e mortalidade e sobre a eficácia do procedimento.

Uma das possíveis complicações da valvoplastia aórtica por cateter-balão encontra-se no acesso arterial femoral clássico. Na tentativa de redução dessa complicação e para preservar a via femoral para procedimentos futuros, realizou-se a dissecção e a punção sob visualização direta da artéria carótida, em substituição à técnica de acesso clássica. Primeiramente tentou-se acesso arterial por punção carotídea percutânea, porém pelas complicações encontradas no primeiro paciente da casuística a estratégia foi mudada para dissecção com punção sob visibilização direta com ótimos resultados e ausência de complicações significativas, com exceção de um caso com sangramento pós-procedimento que necessitou revisão cirúrgica. A via carotídea geralmente permite rápido acesso ao ventrículo esquerdo por encontrar-se em trajeto mais retilíneo, à exceção de casos com inclinação excessiva da aorta ascendente. Interessante ter notado as grandes dimensões da carótida comum, suficientes para a introdução das bainhas, com folga. Especulou-se que o jato de alta velocidade que passa pela valva aórtica cause dilatação não só da aorta ascendente como também da carótida comum. Apesar de não terem sido feitos exames para avaliar a patência da artéria carótida em todos os casos, não houve registro de complicação neurológica, com exceção do primeiro paciente, como já comentado. Ultimamente, passou-se a realizar o exame de Doppler vascular da carótida antes da alta hospitalar. Além disso, tem sido mantida heparina de baixo peso molecular por cerca de 48 horas e ácido acetilsalicílico por três meses após o procedimento. Em um registro europeu com grande número de pacientes, a patência carotídea foi observada em mais de $95 \%$ dos casos durante o seguimento ${ }^{12}$.

Como observado neste estudo, diversas arritmias ventriculares podem ocorrer frequentemente nos pacientes submetidos a valvoplastia, em especial naqueles com disfunção de ventrículo esquerdo. Tal observação denota o quão irritável é o miocárdio desses pacientes e alerta o intervencionista para que esteja sempre preparado para cardioversão elétrica e manobras de ressuscitação durante o procedimento.

Óbitos foram observados em 5 pacientes nesta série, 4 associados ao procedimento $(17 \%)$, a maioria ocorrendo no início do estudo. Tais taxas elevadas também são encontradas na literatura ${ }^{8-10}$ e podem ser explicadas por alguns fatores, sendo a disfunção ventricular previamente presente ao procedimento o principal deles. Além disso, esses pacientes se encontram em tênue equilíbrio em relação à pressão de perfusão coronária (pressão diastólica da aorta - pressão diastólica final do ventrículo esquerdo) pela redução das pressões da aorta e pelo aumento das pressões ventriculares. Por isso, a indução anestésica pode comprometer a perfusão miocárdica se houver hipotensão significativa. Ressalta-se também a necessidade de manipulação cuidadosa do fio-guia para a aorta ascendente após punção sob visualização direta da carótida, a fim de que não resulte em lesões coronárias, como observado em um paciente desta série. Por outro lado, a valvoplastia aórtica foi realizada em paciente com $1,1 \mathrm{~kg}$ nesta casuística sem intercorrências, denotando a factibilidade do método mesmo no mais grave dos pacientes. 
Costa RN, et al. Valvoplastia por Via Carotídea na Estenose Aórtica do Neonato e Lactente Jovem: Resultados Imediatos em Serviços de Referência. Rev Bras Cardiol Invas. 2009;17(4):526-32.

Outro fator que merece ser discutido é a ocorrência ou piora de insuficiência aórtica após o procedimento, como observado nesta casuística. Em um estudo realizado em Boston com 113 neonatos, não foram encontrados fatores de risco para aumento do grau de insuficiência aórtica relacionados com o procedimento ${ }^{9}$. Nesse mesmo estudo, notou-se a existência de progressão da regurgitação aórtica, considerando-se que em seguimento de dez anos 39\% dos pacientes apresentaram insuficiência aórtica moderada ou grave ${ }^{9}$. Nesta casuística, na avaliação pós-valvoplastia, 10 pacientes apresentaram insuficiência aórtica, nenhum com necessidade de intervenção cirúrgica, dados que se repetem na literatura ${ }^{8}$. Nestes pacientes a relação balão-anel variou entre 0,8 e 0,9 e aparentemente não houve nenhum fator de risco associado a essa ocorrência, a despeito do número limitado de pacientes. Não foram utilizadas medidas para redução do débito cardíaco (adenosina ou marca-passo) durante a insuflação do balão, porque a via carotídea promove maior estabilidade ao balão e também porque tais medidas podem provocar arritmias ou assistolia em um miocárdio já suscetível. Especula-se que a indução ou piora da insuficiência aórtica deva-se a anormalidades anatômicas de maior gravidade já presentes nessa faixa etária, incluindo espessamento e assimetria valvular e fusão comissural. Ou seja, o substrato anatômico nessas crianças de menor idade e baixo peso já é desfavorável por natureza. No estudo publicado por Lofland et al. ${ }^{7}$ comparando os resultados da valvotomia cirúrgica e da valvoplastia aórtica, a ocorrência de insuficiência aórtica após o procedimento foi discretamente maior no grupo percutâneo, atingindo significância estatística limítrofe.

Em todos os pacientes deste estudo, houve queda significativa dos gradientes transvalvares por cateterismo e por ecocardiografia. Essa boa resposta ao procedimento se reflete, a curto prazo, na melhora da função ventricular esquerda observada nestes pacientes. A queda das pressões do ventrículo esquerdo e o incremento das pressões da aorta também têm impacto positivo na perfusão coronária, o que pode minimizar a progressão da fibroelastose, característica de miocárdios que sofrem injúrias por redução crônica da perfusão coronária.

Apesar de os resultados de seguimento não terem sido o principal objetivo deste estudo, encontram-se na literatura dados encorajadores. A mortalidade praticamente só está presente logo após o procedimento e estima-se que em dez anos cerca de 50\% dos pacientes estejam livres de reintervenção cirúrgica ou percutânea. Além disso, esses pacientes apresentam crescimento das estruturas esquerdas, em especial da aorta e do ventrículo esquerdo ${ }^{9,10}$.

Este estudo apresenta algumas limitações decorrentes da coleta de dados de maneira retrospectiva, gerando, dessa forma, perda de informações, em especial quanto aos resultados de seguimento. Infeliz- mente, a falta de acompanhamento ambulatorial de pacientes vindos de outras regiões e que retornam para seus locais de origem após a alta hospitalar ainda é uma realidade em nosso meio.

\section{CONCLUSÕES}

Utilizar a valvoplastia percutânea como método de escolha para tratamento paliativo desses pacientes oferece maior simplicidade (em centros com experiência para realização de tais procedimentos), boa relação custo-benefício, menor tempo de internação hospitalar e, também, preservação do tórax e das artérias femorais para possíveis (e talvez inevitáveis) procedimentos futuros. Nesse sentido, se houver necessidade de reintervenção cirúrgica (troca valvar ou cirurgia de Ross), a ausência de aderências intratorácicas pode contribuir para minimizar possíveis complicações.

Apesar das dificuldades da valvoplastia aórtica com cateter-balão geralmente encontradas nesse grupo de baixa faixa etária e alto risco, acredita-se que, quando ultrapassada a curva de aprendizado técnico, o método é relativamente seguro e muito eficaz para redução do gradiente transvalvar, podendo ser considerado de escolha para a paliação inicial de tais pacientes, postergando eventual procedimento cirúrgico.

\section{CONFLITO DE INTERESSES}

Carlos A. C. Pedra é consultor da empresa Bioassist e representante da NuMed, fabricante dos cateteresbalão TyShak utilizados em alguns dos procedimentos. Os demais autores declararam inexistência de conflito de interesses.

\section{REFERÊNCIAS BIBLIOGRÁFICAS}

1. Castañeda AR, Jonas RA, Mayer JE, Hanley FL. Obstruction of the left ventricular outflow tract. In: Castañeda AR, Jonas RA, Mayer JE, Hanley FL, editors. Cardiac surgery of the neonate and infant. Philadelphia: WB Saunders; 1994. p. 315-32.

2. Dyck JD, Freedom RM. Aortic stenosis. In: Freedom RM, Benson LN, Smallhorn JF, editors. Neonatal heart disease. London: Springer Verlag; 1992. p. 357-74.

3. Medeiros Sobrinho JH, Fontes VF, Pontes Jr SC. Estenose e insuficiência aórtica congênitas. Túnel aórtico ventricular esquerdo. In: Medeiros Sobrinho JH, Fontes VF, Pontes Jr SC, editores. Cardiopatias congênitas. São Paulo: Sarvier; 1990. p. 435-55.

4. Schneider DJ, Moore JW. Aortic stenosis. In: Allen HD Driscoll DJ, Shaddy RE, Feltes TF, editors. Moss and Adams' heart disease in infants, children and adolescents including the fetus and young adult. 7th ed. Baltimore: Williams \& Wilkins; 2008. p. 968-87.

5. Justino H, Pedra CAC, Freedom RM, Benson LN. Congenital aortic valve stenosis or regurgitation. In: Freedom RM, Yoo SJ, Mikailian H, Williams WG, editors. The natural and modified history of congenital heart disease. New York: Blackwell; 2004. p. 138-68.

6. Louis JD, Jaggers J. Left ventricular outflow tract obstruction. In: Nichols DG, Cameron DE, Greeley WJ, Lappe DG, Ungerleider RM, Wetzel RC, editors. Critical heart disease in infants and children. St Louis: Mosby; 2006. p. 609-23. 
Costa RN, et al. Valvoplastia por Via Carotídea na Estenose Aórtica do Neonato e Lactente Jovem: Resultados Imediatos em Serviços de Referência. Rev Bras Cardiol Invas. 2009;17(4):526-32.

7. Lofland GK, McCrindle BW, Williams WG, Blackstone EH, Tchervenkov Cl, Sittiwangkul R, et al. Critical aortic stenosis in the neonate: a multi-institutional study of management, outcomes, and risk factors. Congenital Heart Surgeons Society. J Thorac Cardiovasc Surg. 2001;121(1):10-27.

8. Zeevi B, Keane JF, Castañeda AR, Perry SB, Lock JE. Neonatal critical valvar aortic stenosis. A comparison of surgical and balloon dilation therapy. Circulation. 1989;80(4):831-9.

9. McElhinney DB, Lock JE, Keane JF, Moran AM, Colan SD. Left heart growth, function, and reintervention after balloon aortic valvuloplasty for neonatal aortic stenosis. Circulation. 2005;111(4):451-8.
10. Han RK, Gurofsky RC, Lee KJ, Dipchand Al, Williams WG, Smallhorn JF, et al. Outcome and growth potential of left heart structures after neonatal intervention for aortic valve stenosis. J Am Coll Cardiol. 2007;18;50(25):2406-14.

11. Pedra CAC, Esteves CA, Braga SLN, Kambara A, Moreira S, Fontes VF. Carotid approach for interventional procedures in selected patients with congenital heart disease. Rev Bras Cardiol Invasiva. 1997;5(1):16-23.

12. Robinson BV, Brzezinska-Rajszys G, Weber HS, Ksiazyk J, Fricker FJ, Fischer DR, et al. Balloon aortic valvotomy through a carotid cutdown in infants with severe aortic stenosis: results of the multi-centric registry. Cardiol Young. 2000;10(3):225-32. 